\title{
Antimicrobial sensitivity patterns of Staphylococcus species isolated from mobile phones and implications in the health sector
}

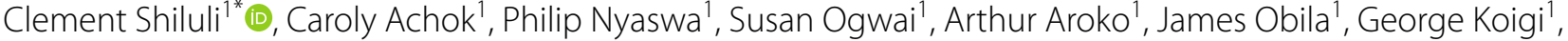 \\ Mustafa Ridhwana', Bildad Okwayo', Dorcas Wanjiru', Linda Lukeba², Eline Ryckaert² ${ }^{2}$ Arne Van Durme², \\ Verena Walschaerts ${ }^{2}$ and Vicky De Preter $^{2}$
}

\begin{abstract}
Objectives: The aim of this research was to determine drug sensitivity profiles of Staphylococcus species isolated from mobile phones of students in Microbiology and Biomedical Laboratory Sciences from UZIMA University, Kisumu (Kenya) and the University Colleges Leuven-Limburg, Leuven (Belgium), respectively.

Results: All mobile phones (16/16, 100\%) had gram-positive bacteria. 3/8 (37.5\%) mobile devices had Staphylococcus aureus. 2/3 (67\%) Staphylococcus aureus strains were resistant to ampicillin, oxacillin, ceftazidime, vancomycin and amoxicillin. Guidelines for disinfection of mobile phones need to be developed urgently to stop transmission of resistant bacteria.
\end{abstract}

Keywords: Staphylococcus species, Gram-positive bacteria, Drug-resistant bacteria

\section{Introduction}

In 2019, approximately 5 billion people had mobile phones worldwide [1]. Health care workers $(\mathrm{HCW})$ use mobile phones to share X-rays, laboratory reports and electrocardiograms. This improves the quality of care particularly during emergencies [2, 3]. Even though the usage of mobile phones in hospitals has a lot of benefits, it is still a large source of contamination. This is because when in use, mobile phones generate heat, which provides suitable conditions for the replication of bacteria present on them. In a 2012 study in Croatia, samples were collected from 50 and 60 mobile phones from $\mathrm{HCW}$ and 60 medical students, respectively. Out of the 110 mobile phones about $35 \%$ were contaminated with one type of microorganism, $28 \%$ with two types and $15 \%$ with three types. According to the results, the most commonly

\footnotetext{
*Correspondence: clementshiluli@gmail.com

1 Department of Microbiology, UZIMA University, Kisumu, Kenya

Full list of author information is available at the end of the article
}

isolated microorganisms were coagulase negative staphylococci and Staphylococcus aureus (S. aureus) (26\%) [2]. The second most abundant bacteria were $S$. aureus with $50 \%$ of the colonies being methicillin-resistant [4]. Normally, S. aureus is found as a commensal bacterium on the skin and in the upper respiratory tract. A wide range of infections can be caused by pathogenic strains of $S$. aureus [5]. Most frequently it causes skin infections, which can manifest in different forms such as boils, folliculitis, cellulitis or even more invasive soft tissue infections. Due to the vastly changing nature of $S$. aureus, a lot of research is needed, particularly in the field of nosocomial infections [6]. The most researched strain is methicillin-resistant $S$. aureus (MRSA), which has become a global health concern over the last couple of years [7]. Infections caused by these multi-drug resistant grampositive organisms are a worldwide problem in hospitals and are a general health hazard to the population since they require a specific type of antibiotic for treatment. The purpose of this study was to isolate $S$. aureus from 
mobile devices of undergraduate students from the University Colleges Leuven-Limburg, and UZIMA University and determine the antimicrobial sensitivity patterns of isolated pathogens.

\section{Main text}

Most microorganisms found in this study, were grampositive bacteria that can easily survive on or are transferred by hands. Bacteria such as $S$. aureus isolated on mobile devices can survive for a long period in a dehydrated environment such as the skin of the hands, the surface on mobile phones and medical devices.

\section{Methods}

This research was done at UZIMA University after obtaining approval from the Institutional Review Board. UZIMA University is located in Kisumu County, in Western Kenya. The University offers undergraduate courses in various disciplines such as Medicine, Nursing, Clinical medicine, Microbiology, Health records and Community health.

An experimental study design was adopted where a total of 16 mobile phones were randomly selected from students. The primary outcome was to isolate Staphylococcus aureus from surfaces of mobile phones. These phones were obtained from students of Microbiology and Biomedical Laboratory Sciences from UZIMA University, Kisumu (Kenya) and the University Colleges LeuvenLimburg, Leuven (Belgium), respectively. The research was part of an annual international project between the two academic institutions to enhance student linkages and experience on practical aspects in the field of microbiology.

Swabs from surfaces of mobile phones were collected using disposable sterile cotton swabs moistened with sterile normal saline.

Approximately $1.84 \mathrm{~g}$ of nutrient agar (Fisher Scientific, Leicestershire, UK) was weighed and dissolved in $80 \mathrm{ml}$ of distilled water in a glass beaker. The glass beaker was heated until the agar was completely dissolved. The media was sterilized by autoclaving at $121{ }^{\circ} \mathrm{C}$ for $15 \mathrm{~min}$. Mobile swabs were plated onto nutrient agar and incubated at $37{ }^{\circ} \mathrm{C}$ for $24 \mathrm{~h}$. After incubation, the colonies were identified by their characteristic appearance on nutrient agar.

Gram staining was used for differentiation of grampositive and gram-negative bacteria by microscopy. Smears were made on microscopic slides from the colonies. The smears were air-dried and heat fixed and flooded with crystal violet (Fisher Scientific, Leicestershire, UK) staining reagent for $1 \mathrm{~min}$. The slides were then washed gently in running water for $2 \mathrm{~s}$. The slides were then flooded with gram's iodine (Fisher Scientific,
Leicestershire, UK) for $1 \mathrm{~min}$ and thereafter washed in a gentle and indirect stream of tap water for $2 \mathrm{~s}$. The slides were decolorized for $15 \mathrm{~s}$ with acetone (Fisher Scientific, Leicestershire, UK) and counterstained with safranin (Fisher Scientific, Leicestershire, UK) for $1 \mathrm{~min}$. The slides were finally washed in running water and blotted on an adsorbent filter paper.

The catalase method was used to differentiate Staphylococci from Streptococci bacteria. Briefly, a small amount of bacterial colony was added to a glass using a sterile loop. A drop of $3 \% \mathrm{H}_{2} \mathrm{O}_{2}$ (Fisher Scientific, Leicestershire, UK) was then added onto the slide and mixed with the colony.

The coagulase method was used as a confirmatory test for Staphylococcus aureus. A drop of staphylococcal colony was emulsified on a glass slide. A sterile wire loop was dipped into undiluted plasma and then emulsified onto the staphylococcal suspension. Coarse clumping was confirmatory for Staphylococci aureus.

The Kirby-Bauer disc diffusion susceptibility test method was used to determine the sensitivity or resistance of bacteria to antibiotics. Mueller-Hinton agar (Fisher Scientific, Leicestershire, UK) was prepared by weighing $1.84 \mathrm{~g}$ of agar in $80 \mathrm{ml}$ of distilled water. The media was briefly heated on a Bunsen burner flame to dissolve and autoclaved as previously described. The media was then dispensed on agar plates and left to solidify. An inoculum was prepared as follows: A sterile inoculating loop was used to pick colonies of the test organism and suspended in $2 \mathrm{ml}$ sterile saline in a tube. A sterile swab was dipped into the inoculum tube and rotated against the side of the tube. The dried surface of the Mueller-Hinton agar plate was inoculated by streaking the swab three times over the entire surface. The antimicrobial impregnated disks (Sigma-Aldrich, Missouri, USA) were placed on the surface of the agar plates using a pair of sterile forceps. The plates were placed inverted in an incubator for $24 \mathrm{~h}$. The zones of inhibition were measured in $\mathrm{mm}$ and the results were scored as resistant, intermediate or susceptible based on the Clinical Laboratory Standards Institute Performance Standards for Antimicrobial Disk Susceptibility Tests.

\section{Results}

Gram-positive bacteria were isolated from all the mobile phones used in this study $(n=16)$. These bacteria were grouped into three groups according to the morphological features after gram staining procedure, rod shaped $(n=8)$, cocci $(n=6)$ and mixed $(n=2)$ (rod and cocci) as shown in Fig. 1.

Only the cocci and the mixed species were further subjected to the catalase test $(n=8)$ to identify the 


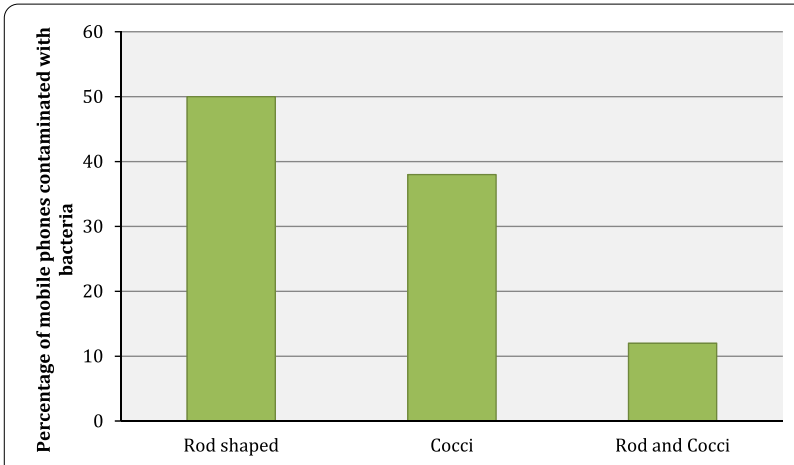

Fig. 1 The morphological forms isolated from mobile phones used in this study were classified into three categories, rods, cocci and both rod and cocci

Staphylococci species. All the samples tested were catalase positive.

Coagulase negative Staphylococcus species were isolated in 5/8 (62.5\%) and 3/8 (37.5\%) mobile devices had Staphylococcus aureus as shown in Fig. 2.

Two out of three (67\%) Staphylococcus aureus strains were resistant to the following antibiotics; ampicillin, oxacillin, ceftazidime, vancomycin and amoxicillin and $1 / 5(20 \%)$ coagulase negative streptococcus was resistant to amoxicillin as shown in Fig. 3.

\section{Discussion}

Globally, there are increasing cases of bacterial infection in humans. This is mainly attributed to factors such as contamination of surfaces of mobile phones that eventually become fomites spreading pathogenic organisms particularly by hands.

Studies on the extent of bacterial colonization of mobile phones will ensure adoption of preventive measures such as disinfection procedures, establishing of mobile phone free zones in hospitals, designing replaceable devices that

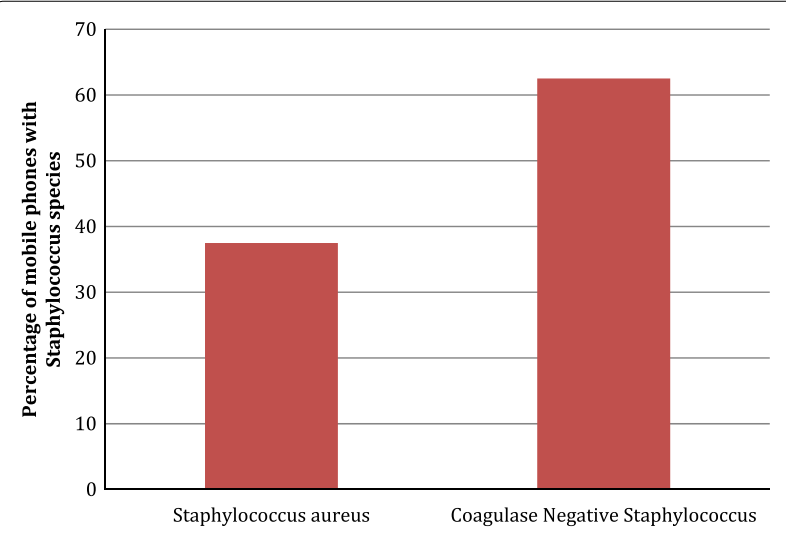

Fig. 2 The coagulase test was used to identify Staphylococcus aureus from coagulase negative Staphylococcus

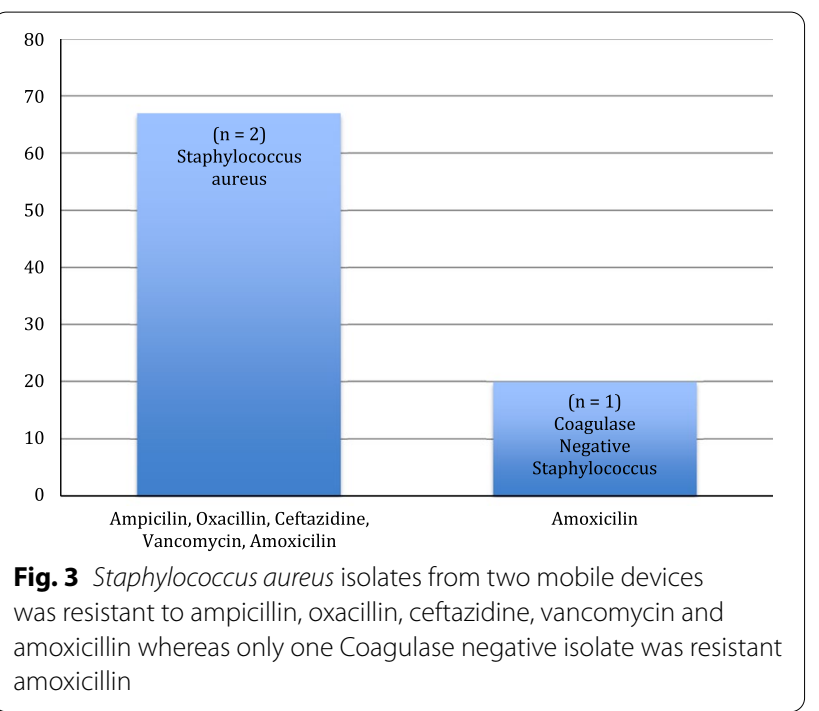

contain less bacterial contamination and making people aware of the current situation about AMR. Once instituted, these measures will reduce the emergence of antimicrobial resistant isolates and nosocomial infections.

All phones used in this study showed significant microbial growth. Our research reported 50\% (8/16), grampositive rod shaped bacteria on mobile phones. This observation is similar to a study that was done in New Zealand at the Fiji National University. In this study, 82\% of mobile phones that were sampled had gram-positive bacillus bacteria [5]. Studies have confirmed that sporeforming gram-positive rods, such as Clostridia, present on mobile devices can be transferred into human food and cause illness once ingested [6]. Our findings, however, are in contrast to a previous study in Slovakia that investigated the effectiveness of mobile phone disinfection in the microbiology laboratory [7]. In this study, 36\% of mobile phones sampled were contaminated with Bacillus bacteria [7]. The reason for this discrepancy could be in the varying sample sizes between the Slovakian study and our research. Comparing the results between the Belgian phones and the Kenyan phones is difficult, because only one sample of $S$. aureus from each country was used in antimicrobial susceptibility test. This is not enough to make a correct conclusion about the difference in resistance between Kenya and Belgium.

In this research, Coagulase negative Staphylococcus (CoNS) and S. aureus accounted for 62.5\% (5/8) and $37.5 \%(3 / 8)$ of the gram-positive cocci bacteria present on the mobile phones, respectively. A recent meta-analysis review in 2020 on previous studies on common bacterial isolates from mobile phones of health care workers confirmed that $66 \%$ of mobile phones were contaminated with CoNS [8]. The same paper also reported that $91 \%$ 
of the phones were colonized with S. aureus [8]. CoNS are commensals of the human skin and mucosal surfaces and cause infections mostly in neonates and immunocompromised individuals [9]. Species include S. hominis, S. capitis, S. warneri, S. epidermidis, and S. haemolyticus. S. epidermidis is the normal microbiota of the nasal surfaces and the newborn umbilicus [9]. S. aureus is an opportunistic bacteria, responsible for nosocomial and community infections [10]. In addition, S. aureus can invade tissues and cause infections such as cutaneous abscesses, endocarditis, and septic shock [10].

Our findings showed that $67 \%$ of the S. aureus isolates were resistant to ampicillin, oxacillin, ceftazidine, vancomycin and amoxicillin. A previous study in Nigeria revealed that $42.8 \%$ of $S$. aureus isolated from mobile phones of non-health care workers was resistant to ampicillin [11]. Clinically, OXACILLIN is used as a marker to screen for mec-A-mediated resistance in Staphylococcus species, therefore, methicillin-resistant isolates are usually identified in culture by using oxacillin break points [12]. Compared to oxacillin susceptible strains, resistant S. aureus strains are slow growers and often show heteroresistance [12]. A past research study reported that methicillin resistant prevalence of $S$. aureus isolated from mobile phones of medical and non-medical staff working in the emergency section of the hospital was $12 \%$ [13]. In Bangladesh, research focused on the presence of multidrug-resistant bacteria on mobile phones of health workers reported that $50 \%$ of the S. aureus isolates were resistant to Ceftazidine [14]. In a study in Saudi Arabia, bacteria was isolated from mobile phones of health care workers and tested against a panel of 12 antimicrobial agents, only one S. aureus isolate had Vancomycin heteroresistance after sensitivity testing [15]. The same study also reported that $38.6 \%$ of the cell phones had been colonized by CoNS. Our research also showed that only one CoNS isolate was resistance to amoxicillin. A meta-analysis research that analyzed 45 studies on the resistance of S. aureus in Ethiopia showed that $77 \%$ of S. aureus were resistant to amoxicillin [16]. There was a higher level of AMR present in the Kenyan sample. The Belgian sample shows a higher susceptibility to penicillin based antimicrobials whereas the Kenyan sample shows complete resistance.

In this study, the most abundant bacteria were the coagulase-negative staphylococci with five colonies and S. aureus was present on three mobile phones. Two S. aureus (one from Kenya and one from Belgium) showed high resistance against a large number of antimicrobials. Additional research on a larger scale is needed for a more in depth analysis. Altogether, the contamination indicates the potential risk of nosocomial infections in hospital environments and indicates the need of measures for the utilization of mobile phone in the health care sector and hand hygiene after usage, to prevent the spread of resistant strains.

\section{Limitations}

Findings from our research should be interpreted with caution because of the small number of mobile phones used. In addition, our research only focused on two types of bacterial species.

\section{Supplementary Information}

The online version contains supplementary material available at https://doi. org/10.1186/s13104-020-05413-7.

Additional file 1: Table S1. Results from gram staining, catalase and coagulase test and the difference between Kenyan and Belgian students. Table S2. Results antimicrobial susceptibility test: inhibition zone comparing standard zone.

Abbreviations

AMR: Antimicrobial resistance; CoNS: Coagulase negative Staphylococcus; HCW: Health care workers; MRSA: Methicillin-resistant S. aureus.

\section{Acknowledgements}

The authors would like to thank Ms Melvin Mbalitsi, Petrov Muthusi (deceased), Gladys Cheptoo, Doreen Mwende, Mary Muthoni, Anne Kahoro, Wema Khandasi, Ernest Murithi and Mwagandi Kahindi.

\section{Authors' contributions}

VDP, CS, CA, PN and SO designed the study and drafted the manuscript. AA and $\mathrm{JO}$ contributed to the data collection. MR, GK, DW and BO performed literature search, review and data analysis. ER, LL, AVD and VW, carried out sample collection, isolation, identification of bacterial isolates, purification, biochemical characterization of bacterial isolates, antimicrobial susceptibility testing, and data interpretation. All authors agree to be accountable for all aspects of the work and approved the final version of the manuscript. All authors read and approved the final manuscript.

Funding

The study did not receive any funding.

Availability of data and materials

All data generated or analyzed during this study are included in this published article (Additional file 1).

\section{Ethics approval and consent to participate}

This study was conducted after approval of the UZIMA University Institutional and Review Board. The study did not involve human or animal subjects.

Consent for publication

Not applicable.

Competing interests

The authors declare that they have no competing interests.

\section{Author details}

${ }^{1}$ Department of Microbiology, UZIMA University, Kisumu, Kenya. ${ }^{2}$ Department of Health, University Colleges Leuven-Limburg (UCLL), Leuven, Belgium.

Received: 27 October 2020 Accepted: 3 December 2020

Published online: 06 January 2021 


\section{References:}

1. https://www.statista.com/statistics/274774/forecast-of-mobile-phone -users-worldwide/. Accessed Sept 2020

2. Kotris I, Drenjančević D, Talapko J, Bukovski S. Identification of microorganisms on mobile phones of intensive care unit health care workers and medical students in the tertiary hospital. Medicinski glasnik. 2017;14(1):85.

3. Ghatole KP. MOBILE PHONES-DO WE NEED DECONTAMINATION? J Evid Based Med Healthc. 2018:5(5):425-8.

4. Trivedi HR, Desai KJ, Trivedi LP, Malek SS, Javdekar TB. Role of mobile phone in spreading hospital acquired infection: a study in different group of health care workers. Natl J Integr Res Med. 2011;2(3):61.

5. National Institutes of Health. Staphylococcal infections. Medline Plus

6. Silva V, Capelo JL, Igrejas G, Poeta P. Molecular epidemiology of Staphylococcus aureus lineages in wild animals in Europe: a review. Antibiotics. 2020;9(3):122

7. Monaco M, de Araujo FP, Cruciani M, Coccia EM, Pantosti A. Worldwide epidemiology and antibiotic resistance of Staphylococcus aureus. In: Staphylococcus aureus 2016. Cham: Springer. pp. 21-56.

8. Olsen M, Campos M, Lohning A, Jones P, Legget J, Bannach-Brown A, McKirdy S, Alghafri R, Tajouri L. Mobile phones represent a pathway for microbial transmission: a scoping review. Travel Med Infect Dis. 2020;28:101704

9. Gleason CA, Juul SE. Avery's diseases of the newborn e-book. Amsterdam: Elsevier Health Sciences; 2017.

10. Lalaouna D, Desgranges E, Caldelari I, Marzi S. MS2-affinity purification coupled with RNA sequencing approach in the human pathogen
Staphylococcus aureus. In: Methods in enzymology, Vol. 612. 2018. Academic Press. pp. 393-411.

11. Nwankwo EO, Ekwunife N, Mofolorunsho KC. Nosocomial pathogens associated with the mobile phones of healthcare workers in a hospital in Anyigba, Kogi state, Nigeria. J Epidemiol Glob Health. 2014;4(2):135-40.

12. Papich MG. Saunders handbook of veterinary drugs. St. Louis: Saunders Elsevier; 2007.

13. Debnath T, Bhowmik S, Islam T, Chowdhury MM. Presence of multidrugresistant bacteria on mobile phones of healthcare workers accelerates the spread of nosocomial infection and regarded as a threat to public health in Bangladesh. J Microsc Ultrastruct. 2018;6(3):165.

14. Safdari H, Aryan E, Sadeghian H, Shams SF, Aganj M. Frequency of methicillin-resistant Staphylococcus aureus (MRSA) in nose and cellular phone of medical and non-medical personnel of emergency departments of Ghaem hospital in Mashhad city. Clin Epidemiol Glob Health. 2020.

15. Banawas S, Abdel-Hadi A, Alaidarous M, Alshehri B, Bin Dukhyil AA, Alsaweed M, Aboamer M. Multidrug-resistant bacteria associated with cell phones of healthcare professionals in selected hospitals in Saudi Arabia. Can J Infect Dis Med Microbiol. 2018;2018.

16. Deyno S, Fekadu S, Astatkie A. Resistance of Staphylococcus aureus to antimicrobial agents in Ethiopia: a meta-analysis. Antimicrob Resist Infect Control. 2017:6(1):85

\section{Publisher's Note}

Springer Nature remains neutral with regard to jurisdictional claims in published maps and institutional affiliations.
Ready to submit your research? Choose BMC and benefit from:

- fast, convenient online submission

- thorough peer review by experienced researchers in your field

- rapid publication on acceptance

- support for research data, including large and complex data types

- gold Open Access which fosters wider collaboration and increased citations

- maximum visibility for your research: over $100 \mathrm{M}$ website views per year

At BMC, research is always in progress.

Learn more biomedcentral.com/submissions 